Revista Española de

I n v e s t i g a c i ón

Crim in ológ i c a

\title{
Información, ¿antídoto frente al "populismo punitivo"?. Estudio sobre las actitudes hacia el castigo de los menores infractores y el sistema de Justicia Juvenil
}

\author{
Eva Aizpurúa González y Esther Fernández Molina ${ }^{1}$
}

Centro de Investigación en Criminología

Universidad de Castilla - La Mancha

Manuscrito recibido el 20 de octubre de 2011 / Publicado el 28 de Noviembre de 2011

\section{RESUMEN}

El estudio de las actitudes públicas hacia el castigo de los menores infractores se ha instituido en uno de los ámbitos de investigación más destacados y prósperos de la Criminología. Sus principales hallazgos han constatado el desconocimiento que caracteriza a la población en lo referente al funcionamiento del sistema de justicia juvenil. Por ello, el presente trabajo tiene como objeto analizar el efecto de la información en las actitudes retributivas y las percepciones relativas a la jurisdicción de menores. Para ello, se ha implementado un diseño cuasi-experimental con grupo de tratamiento y grupo de control, en el municipio de Talavera de la Reina. Los resultados alcanzados, consistentes con la investigación comparada, evidencian que la opinión pública se encuentra supeditada a la cantidad y la calidad de la información de que dispone la comunidad, corroborando que cuanto mayor es el conocimiento ciudadano en esta materia, menores son las actitudes punitivas y los juicios desfavorables hacia el sistema judicial juvenil. Igualmente, los datos resultantes reafirman la naturaleza compleja y multifacética de las actitudes, mediadas por una multiplicidad de factores interdependientes, documentada, con profusión, en las aproximaciones empíricas internacionales.

Palabras clave: actitudes hacia el castigo, delincuencia juvenil, Justicia Juvenil, opinión informada, populismo punitivo.

\footnotetext{
${ }^{1}$ Este trabajo se inserta dentro del proyecto "Percepciones, experiencias y actitudes de los adultos ante el comportamiento antisocial y delictivo de los jóvenes“ (TC2008545) Esther Fernández es IP del proyecto y Eva Aizpurúa realizó el trabajo de campo. Ambas han contribuido en el diseño de la investigación, análisis e interpretación de los resultados, así como en la redacción y la revisión crítica del artículo. Correspondencia: esther.fdez@uclm.es. Las autoras quieren agradecer a las profesoras Rechea Alberola y Bartolomé Gutiérrez su asesoramiento en la realización del análisis y la interpretación de los resultados y las observaciones realizadas por los revisores anónimos de la REIC.
} 


\begin{abstract}
The study of citizens' attitudes towards the punishment of juvenile offenders has been established as one of the most prominent and prosperous spheres of research within Criminology. Its main findings have highlighted the ignorance of the general population towards the way in which the juvenile justice system is run. For this reason, the objective of this project is to analyze the effect of information in compensatory attitudes and perception of juvenile justice system, whilst observing the impact of socio-demographic, personal and instrumental variables. In order to achieve this, a quasi-experimental design has been implemented with a treatment group and a control group, in Talavera de la Reina. The collected results, consistent with the comparative research, show that public opinion is subject to the quantity and quality of the information at the community's disposal. This verifies that as public awareness rises, punitive attitudes and unfavorable opinions against the juvenile justice system, fall. Likewise, the results reaffirm the complex and multifaceted nature of attitudes, influenced by multiple independent factors which are documented in international empirical research.
\end{abstract}

Key words: attitudes towards punishment, juvenile delinquency, juvenile justice, informed opinion, punitive populism.

\title{
I. INTRODUCCIÓN
}

1.1. Las investigaciones sobre actitudes públicas hacia la delincuencia y el sistema de justicia penal en los planos internacional y nacional

Desde que se iniciara la década de 1990 se han sucedido, en numerosos países, múltiples estudios tendentes a conocer las percepciones y actitudes de la población hacia el sistema de justicia penal (entre ellas, Cullen, Evans, Scott \& Burton, 1990; Roberts, 1992; Sprott, 1996; Davis, 1997; Hough \& Roberts, 1998, 2004; Doob, 2000; Anderson, Bromley \& Given, 2005; Haines, 2007; Varona, 2008; Fernández \& Tarancón, 2010) que han erigido esta materia en uno de los principales temas objeto de estudio de la criminología actual. Dicho interés ha emanado de dos fuentes esenciales. La primera de ellas deriva del propio sistema de organización social que sustentamos. En este sentido, la inquietud por estudiar la opinión pública hacia el sistema penal constituye una labor natural y pertinente en el seno de una estructura democrática en la que, en último término, el poder reside en la ciudadanía (Roberts, 2004; Escobar \& Varona, 2007; Mears, Hay, Gertz \& Mancini, 2007). El segundo de estos motivos, por su parte, hace referencia a la vinculación existente entre la opinión ciudadana - ya sea ésta real o supuesta, en tanto que las investigaciones al respecto han sido limitadas hasta períodos recientes - y las decisiones de política criminal impulsadas (Baron \& 
Hartnagel, 1996; Hough \& Roberts, 1998; Díez - Ripollés, 2003; Rechea, Fernández \& Benítez, 2004; Bishop, 2006; Gelb, 2006; Mears et al., 2007).

En concordancia con Medina (2003) gran parte de las determinaciones y reformas adoptadas en el sistema penal no se han fundamentado en un análisis reflexivo y empírico de la realidad social sino que se han desplegado con la pretensión de satisfacer las demandas sociales. De este modo, se ha tratado de responder a lo que Bottoms (1995) designó "populismo punitivo" (citado en Larrauri, 2005), de acuerdo al cual existe una instrumentalización del derecho penal por parte de los gobernantes asentada sobre la asunción de que la ciudadanía exige sanciones más severas hacia la delincuencia (Guetti \& Redlich, 2001), y que los trabajos más actuales han matizado y rebatido (Mathews, 2005; Bishop, 2006; Gelb, 2006; Nagin, Piquero, Scott \& Steinberg, 2006; Scott, Repucci, Antonishak \& Degennaro, 2006; Escobar \& Varona, 2007, Varona, 2008).

En España, el análisis de esta materia constituye una esfera novedosa y escasamente explorada aún, donde la primera investigación de que disponemos data de 2008. Fue Varona (2008) el pionero en el examen de las actitudes de la población hacia el sistema de justicia penal en nuestro contexto, al presentar un estudio piloto, implementado en la ciudad de Girona. Un año más tarde, Ripollés y García (2009), en su estudio de victimación en el marco nacional, recogían información acerca de las actitudes punitivas y el concepto de las instituciones de control penal que atesora la ciudadanía. Posteriormente, Fernández y Tarancón (2010) publicaban una tercera aproximación a este ámbito, ahondando en el conocimiento de las actitudes y percepciones de la población albaceteña hacia la delincuencia juvenil y las respuestas que el sistema preveía para los menores infractores. Finalmente, Marteache, Martínez y Pérez (2010) presentaban un estudio comparativo contrastando la opinión pública y la opinión pública meditada respecto a la delincuencia sexual.

Todo ello permite constatar el carácter novedoso de este ámbito de estudio en España, que contrasta con el alto grado de impulso que el mismo ha detentado en la generalidad de países de nuestro entorno - particularmente en el mundo anglosajón - y que lo ha instituido como uno de los temas angulares de la criminología del siglo XXI. 


\subsection{La investigación de las actitudes ciudadanas hacia la delincuencia juvenil y la jurisdicción de menores}

Si bien es cierto que las investigaciones tendentes a conocer las actitudes de la población hacia el sistema de justicia penal han proliferado notablemente durante los últimos años, la generalidad de los trabajos han examinado la opinión ciudadana en torno a la delincuencia protagonizada por adultos. De este modo, el análisis de la opinión pública hacia la delincuencia juvenil y el tratamiento procurado a los menores infractores constituyen áreas estudiadas en menor medida, donde las tentativas de análisis y evaluación han sido limitadas aún. Esta situación se intensifica en España, donde disponemos, exclusivamente, del estudio desarrollado recientemente por Fernández y Tarancón (2010) y la investigación conducida por García, Martín, Torbey y Rodríguez (2010).

Sin embargo, la escasez de trabajos empíricos que ahonden en esta cuestión no ha sido óbice para que se hayan emprendido numerosas reformas en la Ley Orgánica $5 / 2000$, reguladora de la responsabilidad penal de los menores, (en adelante LO 5/2000), amparándose en las exigencias y demandas ciudadanas. Un ejemplo evidente de esta dinámica lo encontramos en la Exposición de Motivos de la Ley Orgánica 8/2006, modificadora de la LO 5/2000, cuando establece el aumento considerable de los delitos cometidos por menores como motivo de la gran preocupación social y de la pérdida de credibilidad de la Ley por la sensación de impunidad de las infracciones más cotidianas y frecuentemente cometidas por estos menores. Y, en consecuencia, legitima a la judicatura a procurar una mayor proporcionalidad entre la respuesta sancionadora y la gravedad del hecho cometido. Tal y como han evidenciado Fernández y Tarancón (2010), esta última reforma, desplegada ante la ausencia de estudios en materia de actitudes hacia la delincuencia juvenil en España, se fundamenta en tres presupuestos esenciales. En primer lugar, en la existencia de una notable preocupación social en relación al fenómeno de la delincuencia juvenil; en segundo término, en la convicción de que dicha intranquilidad afecta a la credibilidad de la norma, y, finalmente, en la concepción de que una respuesta más punitiva sería, simultáneamente, secundada y deseada por la ciudadanía.

Pese a constituir una temática escasamente estudiada, las investigaciones que se han impulsado en los diversos países permiten delinear ciertas características definitorias de las actitudes ciudadanas con respecto a la delincuencia juvenil y al sistema de justicia de 
menores. Uno de los elementos más destacados que se desprende de la literatura comparada es la tendencia de la población a sobrestimar la magnitud de la delincuencia juvenil, atribuyéndole a ésta un mayor volumen y gravedad de la que en realidad representa (Nacro Youth Crime, 2001; Hough \& Roberts, 2004; Gelb, 2006; Haines, 2007; Fernández \& Tarancón, 2010). Igualmente, existe la propensión a considerar que la delincuencia protagonizada por jóvenes aumenta incesantemente (Hough \& Roberts, 1998, 2004; Haines, 2007; Fernández \& Tarancón, 2010) y que la generalidad de infractores juveniles son reincidentes (Gelb, 2006; Fernández \& Tarancón, 2010). Unida a esta percepción magnificada de la delincuencia perpetrada por menores se encuentra la noción generalizada de que el trato que el sistema les depara es particularmente indulgente (Sprott, 1996; Hough \& Roberts, 1998; Guetti \& Redlich, 2001, Barber \& Doob, 2004; Gelb, 2006; Varma, 2006; Fernández \& Tarancón, 2010). Estas percepciones han sido interpretadas por los autores aludiendo a dos factores esenciales. En primer lugar, haciendo referencia al desconocimiento de la población de los datos de la delincuencia juvenil y del sistema de justicia de menores (Sprott, 1996; Haines, 2007; Fernández \& Tarancón, 2010) y, en segundo término, evocando a los medios de comunicación como agentes fundamentales de información y conformación de la opinión pública que ofrecen una realidad criminal distorsionada (Baron \& Hartnagel, 1996; Hough \& Roberts, 2004; Goidel, Freeman \& Procopio, 2006).

Pese a estas consideraciones, que parecieran augurar una población punitiva, la investigación en la materia ha hallado un amplio apoyo hacia la rehabilitación de los menores (Nagin et al., 2006; Varma, 2006; Krisberg \& Marchiona, 2007; Applegate, Davis \& Cullen, 2009) hacia el tratamiento diferenciado de éstos respecto a los adultos (Baron \& Hartnagel, 1996; Scott et al., 2006), así como hacia la aplicación de penas alternativas al internamiento (Doob, 2000; Hough \& Roberts, 2004; Gelb, 2006). Y ello es así, en tanto que los estudios más recientes han concluido que las actitudes de la población hacia las sanciones no son unidimensionales, sino que, con frecuencias, éstas son flexibles y contradictorias (Bouley \& Wells, 2000; Hutton, 2005; Fernández \& Tarancón, 2010), de modo que el público avala estrategias múltiples de forma simultánea, venciendo la dicotomía educación-castigo instituida en la esfera académica (Cullen, Pealer, Fisher, Applegate \& Santana, 2002; Doble, 2002; Bishop, 2006). 


\subsection{El impacto de la información en las actitudes de la ciudadanía}

Además de los rasgos y características definitorias de las actitudes ciudadanas que se han ido delineando paulatinamente, con la acumulación de evidencia científica, las incipientes investigaciones que se han conducido en el panorama internacional han trascendido la descripción de estos posicionamientos y han ahondado en la incidencia de la información sobre las actitudes y opiniones de la población.

Así, los hallazgos que se desprenden de estos trabajos han revelado que las opiniones públicas se encuentran condicionadas por la cantidad y la calidad de la información de que dispone la ciudadanía (Chapman, Mirrles - Black \& Brawn, 2002; Hough \& Park, 2002; Roberts, 2004; Salisbury, 2004; Gelb, 2006; Green, 2006; Singer \& Cooper, 2008; Marteache et al., 2010; Quinton, 2011) y han evidenciado cómo la provisión de datos concernientes al funcionamiento de la jurisdicción penal no sólo redunda en un conocimiento más preciso y riguroso del mismo, sino que, paralelamente, determina las actitudes retributivas y la confianza ciudadana en el sistema judicial, suscitando percepciones más positivas hacia el mismo (Chapman et al., 2002, Salisbury, 2004; Singer \& Cooper, 2008; Quinton, 2011). Ahora bien, tales aproximaciones advierten que dicha relación entre las actitudes punitivas y el abastecimiento de información habría de interpretarse como una asociación indirecta, condicionada y mediada por múltiples variables (Chapman et al., 2002; Quinton, 2011).

En este sentido, cabe recalcar la existencia de un sólido cuerpo teórico que ha identificado ciertas variables como catalizadoras de estas actitudes, revelando la vinculación de posicionamientos más punitivos con menores niveles de instrucción (Wu, 2000; Mayhew \& Van Kesteren, 2002; Hough \& Roberts, 2004; Scott et al., 2006) con orientaciones ideológicas conservadoras (Baron \& Hartnagel, 1996; Wu, 2000; Scott et al., 2006; Escobar \& Varona, 2007; Mears et al., 2007; Fernández \& Tarancón, 2010), así como con el sexo de los encuestados, desvelando que los varones presentan actitudes más proclives al castigo, que han sido explicadas por los expertos aludiendo a la socialización diferencial de género (Baron \& Hartnagel, 1996; Applegate, Cullen y Fisher, 2002; Scott et al., 2006; Haines, 2007; Mears et al., 2007). La experiencia de victimización, por su parte, ha suscitado resultados contradictorios que oscilan entre aquellos estudios que han hallado una asociación positiva entre la misma y las actitudes punitivas (Anderson et al., 2005; Haines, 2007) y aquellos otros trabajos que han 
encontrado una ausencia de relación entre ambas (Nacro Youth Crime, 2001, Hough \& Roberts, 1999, King \& Maruna, 2009; Wu, 2010).

En nuestro país, éste constituye aún un terreno novedoso y poco cultivado, donde disponemos, únicamente, de la reciente investigación desarrollada por Marteache et al. (2010) en la que se analizan las divergencias entre la opinión pública y la opinión pública meditada en relación a la delincuencia sexual y cuyos resultados son coincidentes con los hallazgos ofrecidos por la literatura comparada. Así, las autoras concluyen que facilitar información a la población motiva el cambio de actitudes en las personas.

Esta revelación es particularmente significativa en la materia que nos ocupa, puesto que uno de los hallazgos más consistentes que se derivan de la investigación, tanto nacional como internacional, es el desconocimiento generalizado de la población acerca del funcionamiento del sistema de justicia penal (Hough \& Roberts, 1998; Doob, 2000; Toharia, 2002; Salisbury, 2004; Varona, 2008), que es aún más agudo cuando se trata de justicia juvenil, como consecuencia de las particularidades que ésta comporta (Sprott, 1996; Hough \& Roberts, 2004; Gelb, 2006; Fernández \& Tarancón, 2010). De este modo, en la medida en que la generalidad de la población no dispone de informaciones directas del sistema de justicia de menores que se deriven de la experiencia propia o vicaria, los medios de comunicación se erigen como principales fuentes informativas en relación a esta temática (Baron \& Hartnagel, 1996; Sprott, 1996; Gelb, 2006; Goidel et al., 2006; Haines, 2007). Así, la ignorancia de los datos verídicos de la delincuencia juvenil y del funcionamiento de la jurisdicción de menores, unida a la representación distorsionada de ambos ofrecida por los medios de comunicación de masas (Rechea et al., 2004; Roberts, 2004; Gelb, 2006; Goidel et al., 2006; Green, 2006; Haines, 2007) pueden estar actuando como catalizadores de las actitudes punitivas ciudadanas.

Como consecuencia de la carencia de estudios que, en el marco nacional, hayan abordado la incidencia e impacto de la información sobre las actitudes públicas hacia la delincuencia juvenil y las sanciones aplicables a los menores infractores, y de acuerdo a la trascendencia que el análisis de las mismas comporta, el presente trabajo responde al doble propósito de contrastar y analizar las divergencias existentes entre la opinión ciudadana informada y desinformada al respecto. 


\section{OBJETIVOS}

\section{a. Objetivo general}

Ahondar en el conocimiento de las actitudes públicas hacia el sistema de justicia juvenil, así como hacia las sanciones aplicables a los menores infractores.

\section{b. Objetivos específicos}

Conocer la incidencia de la información referida a los propósitos, fundamentos y particularidades de la jurisdicción juvenil en la confianza de la población hacia ésta.

Conocer el impacto de la información acerca de las finalidades, principios y características fundamentales del sistema de justicia de menores en las actitudes punitivas de la ciudadanía.

Conocer si el efecto de la información, en caso de hallarse, se encuentra mediado por otras variables demográficas - sexo, edad y nivel de instrucción -, personales ideología política y creencias religiosas - e instrumentales - haber sido víctima de un delito -.

\section{MÉTODO}

\subsection{Diseño de investigación}

El estudio se ha implementado de acuerdo a un diseño cuasi-experimental, con grupo de control estático o no equivalente (García, Ibáñez \& Alvira, 1996; Black, 1999; Clarck - Carter, 2002). En él, la asignación de los sujetos a los grupos de tratamiento y control se ha efectuado empleando un criterio de asignación no aleatoria, distribuyéndolos en forma alterna a uno u otro grupo, habiendo adscrito al primer encuestado al grupo de control.

\subsection{Muestra}

El universo poblacional del trabajo comprende a todos aquellos residentes en el municipio de Talavera de la Reina, que tuvieran la mayoría de edad legal en el momento de realización del trabajo de campo. La muestra se extrajo mediante muestreo no probabilístico por cuotas (Mateo \& García, 1993; Grinnel, 1997) de sexo (varón-mujer) y edad (18-30, 31-43, 44-56, 57-69 y 70 o más), para asegurar la correspondencia de ambos grupos en este sentido y evitar la presencia de divergencias significativas en 
variables sociodemográficas relevantes. La muestra se compuso de 100 sujetos, de ellos, 50 fueron asignados al grupo de control, y 50 fueron adscritos al grupo de tratamiento.

\subsection{Instrumento de medida y variables incorporadas}

El cuestionario aplicado constituye una adaptación del implementado por Fernández y Tarancón (2010), que, a su vez, fue fruto de la traducción y adecuación del instrumento empleado por Hough y Roberts (2004) en el escenario británico. Esta fue la herramienta escogida puesto que constituye el único cuestionario diseñado, específicamente, para cuantificar la opinión pública con respecto al sistema de justicia juvenil, así como las actitudes punitivas ciudadanas, que ha sido aplicado en el contexto español.

Con objeto de incorporar los hallazgos más recientes en la materia, así como de perfeccionar los casos-escenario contenidos en el cuestionario, conforme a las recomendaciones formuladas por las autoras (Fernández \& Tarancón, 2010), se efectuaron ciertas modificaciones en el mismo. Así, se agregaron dos ítems que exploran las experiencias recientes de victimización de los encuestados, tendentes a conocer el impacto de esta variable instrumental sobre las actitudes punitivas ciudadanas. Finalmente, se ha reelaborado cada uno de los casos escenario, de modo tal que su contenido y disposición posibilitaran contrastar las actitudes punitivas de la población en función de la naturaleza de los hechos, de la condición de reincidente del infractor, de las circunstancias psicosociales que acompañan al menor en la comisión del hecho delictivo, así como de su actitud reparadora hacia la víctima.

Así, el cuestionario final consta de un conjunto de variables que articulan cuatro ejes diferenciados. El primero de ellos se compone de un total de diecisiete ítems que tratan de cuantificar el conocimiento y las actitudes públicas hacia la delincuencia protagonizada por menores y adultos. La segunda, explora las actitudes de la ciudadanía hacia el sistema de justicia penal, prestando particular atención al sistema de justicia juvenil. En el tercer apartado, se aplica la técnica del caso-escenario, presentando un total de siete supuestos, en los que se solicita al encuestado que informe, ante hechos delictivos divergentes, qué medida cree que impondría el magistrado y por cuál se inclinaría él, si hubiera de establecer la sanción. Finalmente, el cuarto bloque lo componen las variables socio-demográficas, que constituyen los datos de clasificación. 


\subsection{Manipulación de la variable independiente. La información proporcionada al grupo de tratamiento}

Con objeto de cotejar la existencia de divergencias en las actitudes punitivas de quienes poseen información concerniente al sistema de justicia juvenil frente a quienes no disponen de ella, se elaboró un documento en el que se concretaban, sintéticamente, los principios en que se sustenta la justicia de menores, su naturaleza, las personas sujetas a ella, las diferentes medidas que contempla y los criterios para su aplicación, así como los agentes intervinientes en el proceso penal.

\subsection{Procedimiento}

Una vez efectuadas las modificaciones en el instrumento de medida, y habiendo elaborado el documento con la información a suministrar al grupo de tratamiento, se procedió a realizar una prueba piloto que permitiera verificar la pertinencia de los mismos, con un grupo de diez sujetos, cinco varones y cinco mujeres, pertenecientes a los diferentes grupos de edad predeterminados. Esta prueba inicial posibilitó constatar la suficiencia del cuestionario, y condujo a la introducción de variaciones en el documento informativo, operadas conforme a las limitaciones halladas en este primer ensayo. Por ello, se efectuó una segunda prueba piloto, con estas mismas diez personas, que confirmó su idoneidad.

El procedimiento de recolección de datos se materializó mediante encuesta personal (Rubio \& Varas, 2004), empleando para ello una duración media de veinte minutos en el caso del grupo de control, y de treinta y cinco minutos en el supuesto del grupo de tratamiento. La información que se facilitó a este último grupo fue suministrada verbalmente, de forma previa al pase del cuestionario, acompañando la explicación con la exposición de esquemas y representaciones gráficas e invirtiendo para ello una duración media de trece minutos. Los cuestionarios fueron administrados durante los meses de diciembre de 2010 y enero y febrero de 2011 en diversos espacios de la ciudad, procurando, con ello, reforzar la heterogeneidad de la muestra. 


\subsection{Plan de análisis}

\section{a. Variables dependientes $e$ independientes}

Las variables dependientes empleadas en la articulación del trabajo fueron el conocimiento de la realidad criminal juvenil, la confianza en la jurisdicción de menores y las actitudes punitivas. La primera de ellas fue cuantificada mediante tres ítems, estructurados en forma de escalas de intervalo, que incorporaban la concreción de la proporción de delitos cometidos por menores, respecto de la totalidad de infracciones registradas, la especificación del porcentaje de transgresiones violentas juveniles, así como la determinación de la dimensión de la reincidencia, entre quienes no han alcanzado la mayoría de edad legal. La segunda, fue valorada a través de dos cuestiones, sistematizadas a modo de escalas Likert, que comprendían la valoración del trabajo efectuado por los Juzgados de Menores y la evaluación del trato brindado por éstos y el cuerpo policial a los infractores juveniles. Las actitudes retributivas, por su parte, fueron mesuradas mediante una escala de punición, elaborada partiendo de aquellas variables, recogidas en el cuestionario, que la literatura comparada ha identificado, explícitamente, como punitivas, esto es, el requerimiento de condenas más severas (Doob, 2000; Scott et al., 2006; Varona, 2008, King \& Maruna, 2009), el castigo como propósito principal de las sanciones impuestas (Cullen et al., 2002; Hough \& Roberts, 2004), la preferencia por las medidas privativas de libertad (Kury, Obergfell - Fucks \& Smartt, 2002; Mayhew \& Van Kesteren, 2002) y el rechazo hacia las penas comunitarias, que ha sido identificado por diversos autores como el producto de equiparar éstas con sanciones insuficientemente rígidas (Stead, MacFadyen \& Hasting, 2000; Roberts, 2002; Roberts $\&$ Hough, 2002). De este modo, la escala resultante - que presenta una consistencia interna de $\alpha=, 77$ - está constituida por un total de once ítems, siendo 0 su valor mínimo posible y 11 su puntuación más elevada, indicativa de una mayor preponderancia de actitudes punitivas. 
Tabla 1. Variables dependientes empleadas en el estudio

\begin{tabular}{|cc|c|c|}
\hline VARIABLES DEPENDIENTES & \multicolumn{1}{c}{ AMPLITUD } & X & $\boldsymbol{\sigma}$ \\
\hline Proporción de delitos juveniles & $0-100$ & 41,52 & 20,54 \\
\hline Porcentaje de delitos juveniles violentos & $0-100$ & 54,49 & 23,89 \\
\hline Porcentaje de reincidencia en menores & $0-100$ & 51,38 & 26,36 \\
\hline $\begin{array}{c}\text { Valoración del trabajo desarrollado por los } \\
\text { Juzgados de Menores }\end{array}$ & $1-5$ & & \\
\hline $\begin{array}{c}\text { Valoración de la forma en que la policía y los } \\
\text { juzgados tratan a los menores infractores }\end{array}$ & Muy dura - Muy indulgente & 3,55 &, 806 \\
\hline Escala de punición & $0-11$ & 1,59 & 1,525 \\
\hline
\end{tabular}

En armonía con las observaciones provenientes la investigación empírica internacional (Baron \& Hartnagel, 1996; Wu, 2000; Applegate et al., 2002; Mayhew \& Van Kesteren, 2002; Hough \& Roberts, 2004; Scott et al., 2006, Escobar \& Varona, 2007; Haines, 2007; Mears et al., 2007; Fernández \& Tarancón, 2010) las variables independientes e intervinientes observadas fueron, respectivamente, el tratamiento provisto y los factores socio-demográficos e instrumentales de los encuestados - esto es, el sexo, la edad, el nivel de instrucción, la ideología política, las creencias religiosas y la experiencia de victimación de los participantes -.

Tabla 2. Variables independientes e intervinientes en el estudio

\begin{tabular}{|c|c|c|c|}
\hline V. INDEPENDIENTES & AMPLITUD & $\mathbf{X}$ & $\boldsymbol{\sigma}$ \\
\hline Tipo de grupo & 1 (Control) -2 (Tratamiento) & - & - \\
\hline Sexo & 1 (Mujer) -2 (Hombre) & - & - \\
\hline Edad & $18-89$ & 50,28 & 18,79 \\
\hline Nivel de instrucción & $\begin{array}{c}1 \text { (Carece de estudios básicos) - } 5 \text { (Estudios } \\
\text { superiores) }\end{array}$ & 2,57 & 0,832 \\
\hline Posición ideológica & 0 (Extrema izquierda) - 10 (Extrema derecha) & 5,35 & 1,914 \\
\hline Creencias religiosas & $\begin{array}{c}1 \text { (Se considera creyente) }-2 \text { (No se considera } \\
\text { creyente) }\end{array}$ & - & - \\
\hline $\begin{array}{l}\text { Experiencia de } \\
\text { victimación }\end{array}$ & $\begin{array}{c}1 \text { (Ha sido víctima de un delito) - } 2 \text { (No ha sido } \\
\text { víctima de un delito) }\end{array}$ & - & - \\
\hline
\end{tabular}

\section{b. Tratamiento estadístico de los datos}

El análisis estadístico de los datos, operado utilizando el software SPSS, en su versión 19.0, incorpora análisis descriptivos, pruebas de asociación entre variables así como estadísticos de comprobación de distribuciones entre grupos. De acuerdo a las 
propiedades de las variables, se han empleado contrastes de relación paramétricos y no paramétricos. El análisis de las divergencias entre grupos se condujo mediante las pruebas U de Mann-Whitney y T, ambas para muestras independientes. Finalmente, la exploración y cuantificación de la relación entre las actitudes punitivas y el conjunto de variables predictoras considerado se efectuó mediante análisis de regresión lineal múltiple.

\subsection{Limitaciones y sesgos}

Como consecuencia del diseño de investigación implementado, conforme al cual la asignación de los sujetos a los grupos de tratamiento y control no es aleatoria - y, por ende, no puede garantizarse la equivalencia inicial de uno y otro - la validez interna del experimento es restringida (Heppner, Kivlighan \& Wamplod, 2008; Jackson, 2009). Igualmente, cabe subrayar las limitaciones subyacentes a la existencia de un sesgo de selección (Berger, 2005) en la elección de los sujetos que participaron en la prueba piloto del instrumento, en tanto que las mismas personas que intervinieron en el pase inicial del cuestionario, valoraron las modificaciones inducidas a partir de él, existiendo, así, la amenaza de que las respuestas de esta segunda verificación hayan sido afectadas como consecuencia de su participación en la prueba precedente. No obstante, cabe recordar que estos sujetos no formaron parte de la muestra última del experimento.

Finalmente, la técnica de recogida de datos escogida, pese a haberse consolidado como procedimiento preferente de aproximación al estudio de las actitudes retributivas (Broadhurst, 1982; Roberts, 2004; Gelb, 2006), no se halla exenta de limitaciones referidas a su ineptitud para recoger la complejidad, el dinamismo y la heterogeneidad de las opiniones ciudadanas, ofreciendo una óptica parcial de las mismas (Stalans, 2002; Roberts, 2004; Green, 2006). Con objeto de mitigar tales insuficiencias, el cuestionario implementado incorpora a las preguntas tradicionales, la denominada técnica del casoescenario, tendente a prevenir las respuestas fundadas en estereotipos e imágenes distorsionadas de los infractores, que ha verificado ofrecer una perspectiva más rigurosa de las actitudes públicas (Hough \& Roberts, 1998; Doob, 2000; Stalans, 2002; Bishop, 2006; Applegate et al., 2009; Varona, 2008). 


\section{RESULTADOS}

\subsection{Descripción de la muestra}

Fruto de la forma de muestreo implementada, la muestra es homogénea en cuanto a sexo y grupos de edad. En relación con las restantes variables consideradas y tras haber efectuado las operaciones pertinentes, puede concluirse la inexistencia de divergencias estadísticamente significativas entre los grupos de control y tratamiento, a excepción de la adscripción religiosa, en la se aprecia la presencia de diferencias significativas entre uno y otro, que serán observadas durante la interpretación de los resultados $\left(X^{2}=4,32\right.$; g.l.= $1 ; \mathrm{p} \leq 0,05)$.

Atendiendo a la nacionalidad de los encuestados, hallamos que un $91 \%$ de ellos posee nacionalidad española, frente a un $9 \%$ de los/as mismos, que ostenta nacionalidad extranjera. Estos hallazgos no distan sustancialmente de los datos arrojados por el INE, en los que se estima que la población talaverana que cuenta con nacionalidad española es de un $88,3 \%$ del total ${ }^{2}$.

En relación al nivel de estudios, advertimos que un porcentaje mayoritario de la muestra (46\%) dispone de estudios obligatorios, seguido de una proporción significativa que posee bachillerato (34\%); los encuestados con estudios universitarios constituyen el $14 \%$ del total, mientras que aquellos que no han concluido la enseñanza obligatoria componen el $6 \%$ de la muestra. Confrontando estos datos con los emitidos por el Ministerio de Educación en $2010^{3}$, referidos a la población castellano-manchega, constatamos la ausencia de desviaciones trascendentales.

Respecto a su actividad, observamos que una proporción mayoritaria de los participantes se encuentran ocupados (36\%) y son receptores de prestaciones (37\%), existiendo un igual número $(9 \%)$ de personas desempleadas, estudiantes y trabajadoras domésticas no remuneradas. De conformidad con la clasificación de ocupaciones con que opera el CIS, la generalidad de trabajadores son profesionales, técnicos y similares, seguidos de comerciantes, vendedores y análogos.

\footnotetext{
${ }^{2}$ Obtenidos a partir de la Explotación Estadística del Padrón, referidos a 2010.

${ }^{3}$ Instituto de Evaluación del Ministerio de Educación y Ciencia (2010). Sistema estatal de indicadores de la evaluación.
} 
En cuanto a su posicionamiento ideológico, un $42 \%$ de la muestra se considera conservadora, un tercio se reconoce neutral y un cuarto se define progresista. Finalmente, las tres cuartas partes de los encuestados manifiestan ser religiosos, adscribiéndose, en su mayoría, al catolicismo $(90,7 \%)$.

\subsection{El conocimiento de la población talaverana de los datos de la delincuencia juvenil}

Con la finalidad de analizar el conocimiento que alberga la población en materia de delincuencia juvenil, se articularon cuatro preguntas, tendentes a explorar su percepción acerca de la proporción de delitos consumados por menores y su evolución en el transcurso del tiempo, el porcentaje de delincuencia juvenil violenta, así como la magnitud de la reincidencia en este grupo de infractores, hallándose diferencias estadísticamente significativas entre ambos grupos en esta última variable $(\mathrm{t}=1,994$; g.l.=98; p<0,05). En las dimensiones restantes no se encontraron diferencias representativas a nivel estadístico, aunque sí se advierten desemejanzas entre uno y otro grupo, tal y como se evidencia en los párrafos sucesivos.

Así, hallamos que, aunque ambos grupos estimaron, mayoritariamente, que el volumen de delincuencia juvenil constituye entre el 26 y el $50 \%$ de la criminalidad registrada, un $32 \%$ del grupo de control, frente a un $20 \%$ del grupo de tratamiento, consideraron que una proporción superior a la mitad de los delitos fueron perpetrados por jóvenes.

Igualmente, un porcentaje mayoritario de los encuestados valoró que la delincuencia juvenil se ha incrementado en el transcurso de los dos últimos años, suscribiendo esta premisa un 60,4\% de los integrantes del grupo de tratamiento y un $68 \%$ de los componentes del grupo de control, siendo, en uno y otro caso, su principal fuente de información las noticias provenientes de la prensa, la radio y la televisión (71,1\% y 55,8\%, respectivamente). Concretamente, se considera que las infracciones juveniles que han aumentado en mayor medida han sido los delitos violentos (42,7\% del grupo de tratamiento, y 36,4\% del grupo de control). Dicha asimilación de la delincuencia juvenil con la delincuencia violenta aparece reafirmada, nuevamente, cuando se solicita a los encuestados que valoren la proporción de delincuencia violenta protagonizada por jóvenes. Así, un porcentaje mayoritario del grupo de control (38\%) mantiene que ésta constituye entre el $51 \%$ y el $75 \%$ del conjunto, mientras que el grupo de tratamiento la enclava, en su mayoría (36\%) entre el $26 \%$ y el $50 \%$. 
En lo que a reincidencia respecta, tanto uno como otro grupo consideran que los menores que, tras haber cometido un delito, incurren en otro en el intervalo de un año suponen, entre el $26 \%$ y el $50 \%$ del conjunto, y así lo refrenda el $40 \%$ del grupo de control, y el $38 \%$ del grupo de tratamiento. No obstante, cabe recalcar que un $28 \%$ de los integrantes del grupo de control estima que la reincidencia juvenil se ubica entre el 76 y el $100 \%$ de la totalidad, mientras que esta proporción desciende a un $14 \%$ en el grupo objeto de tratamiento, cuya segunda respuesta más avalada, es que la repetición de conductas delictivas supone entre el $0 \%$ y el $25 \%$ del total.

\subsection{La confianza en el sistema de justicia juvenil}

Con objeto de conocer la confianza depositada en el sistema de justicia juvenil y contrastar el impacto de la información sobre ella, se formularon dos preguntas en las que se solicitaba a los sujetos que valorasen el trabajo efectuado por los Juzgados de Menores, así como el trato brindado por éstos y el cuerpo policial a los jóvenes infractores.

Los resultados hallados evidencian que existen diferencias significativas entre uno y otro grupo en ambos casos (Valoración del trabajo desarrollado por los Juzgados de Menores, U de Mann-Whitney=653.000; $Z=-2,077 ; p=0,038$; Evaluación de la forma en que los Juzgados y la policía tratan a los menores infractores, U de MannWhitney=703.500; $Z=-2,797 ; p=0,005)$. Así, advertimos que la pertenencia al grupo de tratamiento se asocia a una valoración más positiva del trabajo efectuado por los Juzgados de Menores, mientras que la adhesión al grupo de control se vincula a evaluaciones más negativas del mismo $\left(\right.$ rho $\left.=-, 229 ; \mathrm{p}<, 05^{4}\right)$.

\footnotetext{
${ }^{4}$ Habiéndose codificado el tipo de grupo como 1. Control y 2. Tratamiento, y la valoración del trabajo desempeñado por los Juzgados de Menores conforme a una escala Likert en la que 1 simbolizaba "excelente" y 5 representaba "muy malo".
} 
Figura 1. Valoración del trabajo desarrollado por los Juzgados de Menores.

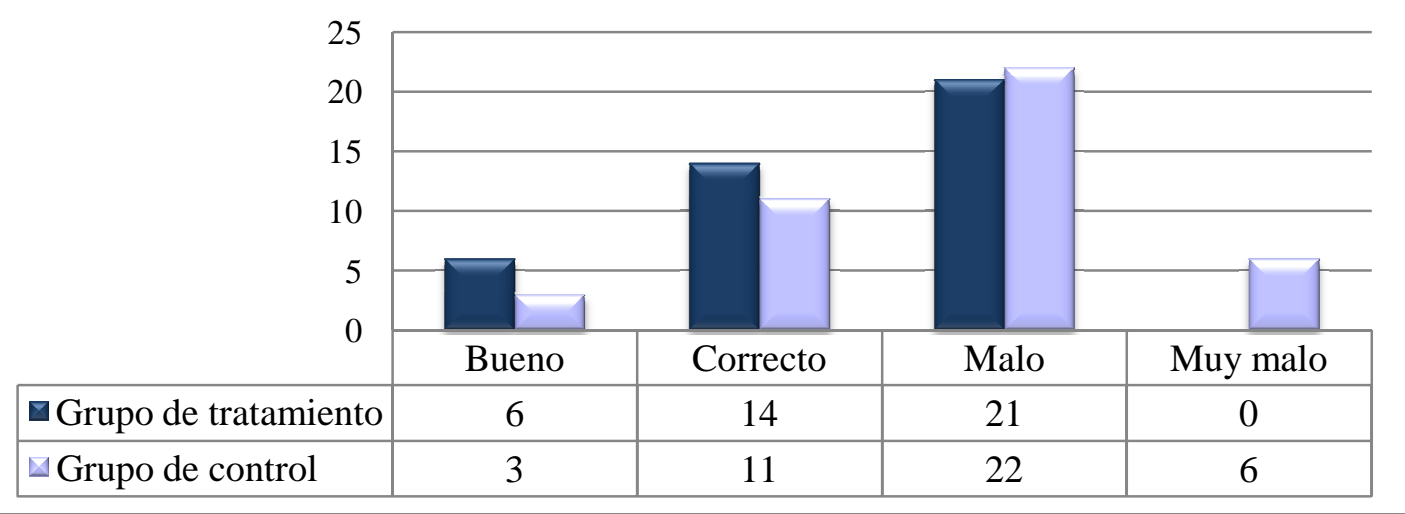

Del mismo modo, hallamos que la calificación como indulgente y muy indulgente del tratamiento procurado a los infractores juveniles de parte de los Juzgados de Menores y el cuerpo policial se encuentra relacionada con la pertenencia al grupo de tratamiento $\left(\right.$ rho $\left.=-, 295 ; \mathrm{p}<, 01^{5}\right)$.

Figura 2. Valoración de la forma en que la policía y los Juzgados tratan a los/as menores infractores/as.

\begin{tabular}{|c|c|c|c|c|c|}
\hline & & & & & \\
\hline 20 & & & & & \\
\hline 15 & & & & & \\
\hline 10 & & & & & \\
\hline 5 & & & & & \\
\hline 0 & & $B$ & & & \\
\hline & Muy dura & Dura & Correcta & Indulgente & $\begin{array}{c}\text { Muy } \\
\text { indulgente }\end{array}$ \\
\hline Grupo de tratamiento & 0 & 3 & 23 & 16 & 0 \\
\hline$\triangle$ Grupo de control & 1 & 2 & 15 & 21 & 10 \\
\hline
\end{tabular}

\subsection{Las actitudes punitivas}

Las actitudes punitivas han sido cuantificadas mediante la escala de punición referida en el plan de análisis, a partir de la cual observamos que, globalmente, las puntuaciones más prominentes se concentran en sus valores más bajos $\left(\bar{X}=1,59 ; \mathrm{M}_{\mathrm{e}}=\right.$ 1), indicativos de una menor preponderancia de éstas - lo cual se hace patente, en la

\footnotetext{
${ }^{5}$ Habiéndose codificado el tipo de grupo como 1. Control y 2. Tratamiento, y la valoración del trato brindado a los infractores juveniles mediante una escala Likert en la que 1 significaba "muy duro" y 5 denotaba "muy indulgente".
} 
medida en que los tres primeros valores albergan el $78 \%$ del porcentaje acumulado de esta variable.

Figura 3. Escala de punición.

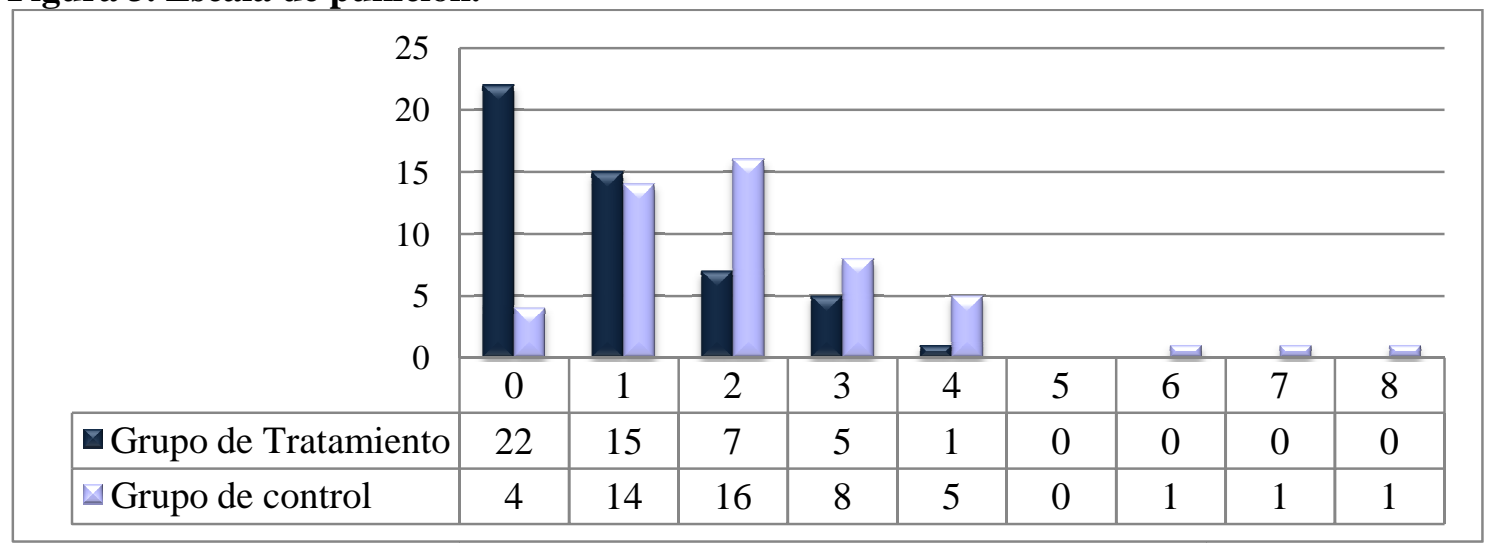

Analizando las simetrías entre grupos, hallamos diferencias estadísticamente significativas entre ellos, tal y como atestiguan los resultados obtenidos $(\mathrm{t}=4,52$; g.l. = 98; $\mathrm{p}=, 000)$, certificándose la existencia de relación entre la pertenencia al grupo de tratamiento y la obtención de una menor puntuación en la escala de punición así como la pertenencia al grupo de control y la consecución de puntaciones más elevadas en la escala $\left(r_{b p}=-0,415 ; p=, 000\right)$.

\subsection{El impacto de las variables demográficas, personales e instrumentales}

Tras conocer las diferencias inter-grupos en las actitudes punitivas, se exploraron la incidencia e impacto del tratamiento, así como de las variables demográficas - sexo edad y nivel de instrucción -, personales - ideología política y creencias religiosas - e instrumentales - experiencia de victimación - sobre las primeras, con objeto de conocer en qué medida las actitudes punitivas se encuentran mediadas por tales factores. Para ello, se efectuó un análisis de regresión por pasos sucesivos, cuyos resultados se exponen en la tabla 4. De ellos se infiere que el conjunto de variables conformado por el tipo de grupo, la edad y las creencias religiosas a las que se adscriben los encuestados explica un $25,9 \%$ de la varianza en la escala de punición, lo cual es indicativo de un tamaño de efecto grande en las investigaciones de esta índole (Cohen, 1998) ${ }^{6}$.

\footnotetext{
${ }^{6}$ Cohen estableció la convención de que aquellos resultados de $\mathrm{R}^{2}$ semejantes a ,02; ,13 y ,26 representaran, respectivamente, la expresión de tamaños de efecto pequeños, medianos y grandes.
} 
En el primer paso, el tipo de grupo fue introducido en la ecuación de regresión, denotando que es el elemento que contribuye en mayor medida al modelo. Concretamente, esta variable dilucida el $16,4 \%$ de la varianza en la variable independiente, y subsecuentemente, representa un tamaño de efecto mediano - grande (Cohen, 1998). Observando la dirección correspondiente a los coeficientes, y en concordancia con los resultados previos, advertimos que el incremento de las actitudes punitivas se vincula a la pertenencia al grupo de control. En el paso sucesivo, la edad fue incorporada a la ecuación, con una capacidad explicativa del 4,4\% de la varianza en las actitudes punitivas, revelando que la menor edad de los encuestados se asocia con una mayor punitividad. Finalmente, en la última fase se agregaron las creencias religiosas a la ecuación de regresión, explicando un 5,1\% de la varianza en las actitudes retributivas, esclareciendo que las personas que poseen creencias religiosas registran valores más elevados en la escala, frente a quienes declaran no adherirse a estos ideales. Combinando el efecto de sendas variables - demográfica y personal -, hallamos que, globalmente, proporcionan un tamaño de efecto de pequeño a mediano (Cohen, 1998).

Tabla 4. Regresión múltiple por pasos sucesivos (variables en la ecuación)

\begin{tabular}{|l|c|c|}
\hline \multicolumn{1}{|c|}{ VARIABLES PREDICTORAS } & $\mathbf{B}^{\mathbf{a}}$ & Beta \\
\hline Tipo de grupo (tratamiento) & $-1,260$ &,$- 415^{* *}$ \\
\hline $\mathbf{R}^{2}$ corregida ,164 & & \\
\hline Tipo de grupo (tratamiento) & $-1,245$ &,$- 410^{* *}$ \\
\hline Edad &,- 018 &,$- 227^{*}$ \\
\hline $\mathbf{R}^{2}$ corregida ,208 & -1.073 &,$- 354^{* *}$ \\
\hline Tipo de grupo (tratamiento) &,- 026 &,$- 321^{* *}$ \\
\hline Edad &,- 923 &,$- 263^{* *}$ \\
\hline Creencias religiosas (presencia de idearios religiosos) & & \\
\hline $\mathbf{R}^{2}$ corregida ,259 & \\
\hline
\end{tabular}

${ }^{\mathrm{a}}$ Coeficientes no tipificados. $\quad *$ Probabilidad $<0,05 ; * *$ Probabilidad $<0,01$

\section{DISCUSIÓN}

\subsection{La percepción distorsionada de la delincuencia juvenil}

En primer lugar, cabe recalcar el desconocimiento generalizado acerca de los datos de la delincuencia juvenil que caracteriza a la población encuestada, y que se intensifica en mayor medida en el grupo de control. Así, advertimos que existe una sobredimensión de la magnitud de este fenómeno, que contrasta con su alcance real. Mientras la 
generalidad de los encuestados estimó que la delincuencia protagonizada por jóvenes constituía entre el $26 \%$ y el $50 \%$ de la criminalidad total, los datos arrojados por las instancias oficiales nos revelan que la proporción de detenidos juveniles integra, exclusivamente, el $6,2 \%$ del conjunto ${ }^{7}$. Unida a esta visión desmesurada de la delincuencia juvenil, la generalidad de los componentes de uno y otro grupo - aunque, nuevamente, el grupo de control en mayor medida -, consideraron que los delitos cometidos por menores se han incrementado recientemente. Y ello, pese a que los datos de que disponemos contradigan tal presunción (Fernández, Bartolomé, Rechea \& Megías, 2009).

En lo que a delincuencia violenta respecta, asistimos a una estimación acertada del grupo de tratamiento, que la sitúa entre el $26 \%$ y el $50 \%$ del conjunto, frente a una percepción particularmente magnificada del grupo de control, que la ubica entre el $51 \%$ y el $75 \%$ de las infracciones totales, y que contrasta con la cifra de $32,04 \%$ proporcionada por los organismos oficiales ${ }^{8}$.

Finalmente, en relación a la reincidencia, advertimos que tanto uno como otro grupo, valoraron, oportunamente, la proporción de jóvenes que, tras haber cometido una infracción, incurren en otra en el intervalo de un año, enclavándola entre un 26 y un $50 \%$ del total, que coincide con los datos ofrecidos por los estudios más recientes conducidos en nuestro contexto, que la cifran entre un 22,7\% y un 30\% (Capdevilla, Ferrer \& Luque, 2005; Bravo, Sierra \& Del Valle, 2009; San Juan \& Ocáriz, 2009).

Esta percepción acrecentada de la delincuencia juvenil es coincidente con los resultados hallados por la investigación comparada (Sprott, 1996; Hough \& Roberts, 1998, 2004; Nacro Youth Crime, 2001; Anderson et al., 2005; Haines, 2007; Halsey \& White, 2008; Fernández \& Tarancón, 2010), y ha sido justificada por los autores aludiendo al desconocimiento de la población de los datos de la delincuencia juvenil y del funcionamiento del sistema penal (Sprott, 1996; Haines, 2007; Minkes, 2007), así como designando a los medios de comunicación como principales protagonistas en la conformación de la opinión pública, que procuran datos distorsionados de la realidad criminal (Baron \& Hartnagel, 1996; Guetti \& Redlich, 2001; Hough \& Roberts, 2004; Goidel et al., 2006; Halsey \& White, 2008). Esta explicación resulta coherente con los

\footnotetext{
${ }^{7}$ En efecto, los datos ofrecidos por el Ministerio de Interior, referidos a 2009, indican que la población total de detenidos fue de 307.730, de los cuales 19.025 fueron menores de edad, de lo que se infiere que la proporción de detenidos juveniles constituye el 6,2\% de la criminalidad registrada.

${ }^{8}$ Nuevamente, Ministerio de Interior, 2009.
} 
resultados mostrados en el presente estudio, puesto que la generalidad de los integrantes de ambos grupos precisaron que las fuentes que avalaban su percepción del incremento de la delincuencia juvenil eran la radio, la prensa y la televisión.

En definitiva, los hallazgos que se desprenden son armónicos con los resultados ofrecidos por la investigación científica internacional que evidencian el desconocimiento de los datos delincuenciales, concretándose en una sobredimensión de las cifras criminales (Barber \& Doob, 2004; Hough \& Roberts, 2004; Gelb, 2006; Haines, 2007; Halsey \& White, 2008; Fernández y Tarancón, 2010).

Finalmente, destaca la existencia de diferencias inter-grupos, que alzan al grupo de tratamiento como conocedor más riguroso de la delincuencia desplegada por menores, pese a que la información suministrada no incorporaba referencias a este respecto y que sugiere que el conocimiento referido al funcionamiento de la jurisdicción juvenil, puede actuar como promotor de nociones más realistas y precisas en esta materia.

\subsection{La información, catalizadora de la confianza en la jurisdicción juvenil}

En consonancia con los hallazgos resultantes de la investigación comparada (Baron \& Hartnagel, 1996; Hough \& Roberts, 1998, 2004; Toharia, 2002; Barbel \& Doob, 2004; Scott et al., 2006; Varma, 2006; Krisberg \& Marchiona, 2007; Fernández \& Tarancón, 2010), el estudio revela una percepción eminentemente negativa hacia los agentes de control social formal intervinientes en el sistema de justicia juvenil, que se materializa en una valoración desfavorable del trabajo efectuado por los Juzgados de Menores así como en la noción de un trato particularmente benevolente brindado por éstos y el cuerpo policial a los delincuentes juveniles. Percepciones ambas que se intensifican en el grupo de control, existiendo diferencias significativas entre ambos grupos, que evidencian que la provisión de información referida al funcionamiento del sistema de justicia juvenil estimula la confianza depositada en él.

De este modo, la información desempeñó un rol angular en la modificación de las actitudes de los encuestados hacia los agentes de control social formal que participan del sistema judicial de menores, que es concordante con los resultados arrojados por la investigación internacional. Resultados que constatan la maleabilidad de las actitudes ciudadanas, las cuales fluctúan en una dirección menos retributiva en respuesta a los datos disponibles (Davis, 1997; Nacro Youth Crime, 2001; Hough \& Park, 2002; 
Indermaur \& Hough, 2002), así como la forma en que el desconocimiento de la población a este respecto obstaculiza su confianza en el sistema (Chapman et al., 2002; Mirless - Black, 2002; Green, 2006), lo que nos conduce a erigir la provisión de información como resorte esencial en la transformación de las percepciones y actitudes ciudadanas.

\subsection{La información, factor de protección frente a las actitudes punitivas}

A priori, cabe indicar que la muestra analizada constituye una población que podríamos definir como poco punitiva a tenor de los datos, los cuales revelan que, considerando ambos grupos, quienes han obtenido una puntuación de 0 y 1 en la escala de punición, y, consecuentemente, han registrado actitudes poco o nada punitivas constituyen el 55\% de la muestra global. Resultados que avalan los hallazgos dimanantes de un creciente cuerpo de investigación que han revelado que las actitudes de la ciudadanía no son tan punitivas como se ha tendido a aceptar, y que han matizado el supuesto anhelo retributivo de la población (Allen, 2004; Maruna \& King, 2004; Roberts, 2004; Matthews, 2005; Bishop, 2006; Gelb, 2006; Scott et al., 2006; Escobar \& Varona, 2007; Minkes, 2007; Varona, 2008; Indermaur, 2009).

No obstante, este rasgo global se encuentra mediado por la información de que disponen los encuestados, tal y como evidencian las diferencias, estadísticamente significativas, existentes entre el grupo de control y el grupo de tratamiento, que constatan que la muestra desinformada presenta actitudes sustancialmente más punitivas que la muestra informada.

Todo ello permite reafirmar la maleabilidad y flexibilidad de las actitudes hacia el castigo, documentada en una multiplicidad de estudios recientes (Hough \& Park, 2002; Indermaur \& Hough, 2002; Mirrless - Black, 2002; Hutton, 2005), así como la capacidad de la información para mitigar las actitudes punitivas que alberga la población, erigiéndose como una estrategia de especial trascendencia, impulsora de posiciones menos retributivas y de percepciones positivas hacia el sistema de justicia juvenil (Baron \& Hartnagel, 1996; Davis, 1997; Nacro Youth Crime, 2001; Cullen et al., 2002; Mirrless - Black, 2002; Roberts \& Hough, 2002; Allen, 2004; Gelb, 2006).

Tal y como se adelantaba en la introducción, nuestra LO 5/2000 ha sufrido diversas reformas desde su promulgación que han incrementado, gradualmente, el carácter 
retributivo de la justicia de menores, y que han sido justificadas por el legislador amparándose en las demandas ciudadanas (Exposición de Motivos, LO 15/2003, Exposición de Motivos, LO 8/2006). Sin embargo, los resultados que se desprenden del presente estudio, así como aquellos otros alcanzados por Fernández y Tarancón (2010) y García et al. (2010) - que constituyen las únicas investigaciones que se han conducido en nuestro contexto en materia de actitudes hacia el castigo de los menores infractores -, evidencian que esta respuesta legislativa no refleja con exactitud y precisión la voluntad pública en lo que respecta al tratamiento de éstos. No obstante, esta paradoja no constituye una tendencia exclusiva de nuestro marco legislativo, sino que ha sido profusamente documentada y litigada en los trabajos enmarcados en el escenario anglosajón (Matthews, 2005; Bishop, 2006; Nagin et al., 2006; Lynne \& Indermaur, 2007).

\subsection{El rol desempeñado por las variables demográficas, personales e instrumentales}

Corroborado el efecto de la información en las actitudes punitivas, se exploró su magnitud, analizándose, así mismo, el impacto de las variables socio-demográficas, personales e instrumentales sobre las primeras. Los datos resultantes desvelan que el tratamiento, por sí mismo, tiene una capacidad explicativa de un 16,4\% de la varianza en la escala de punición, representando un tamaño de efecto de mediano a grande en las investigaciones de esta naturaleza (Cohen, 1998), mientras que la edad y las creencias religiosas a que se adscriben los encuestados explicaron, colectivamente, un 9,5\% de la varianza en las actitudes retributivas, revelando un tamaño de efecto de pequeño a mediano (Cohen, 1998).

Estos hallazgos son coherentes con los derivados de la investigación comparada, que han instituido la información como procedimiento medular para aminorar las actitudes punitivas de la ciudadanía (Chapman et al., 2002; Hough \& Park, 2002; Mirrless - Black, 2002; Roberts, 2004), pero que han evidenciado que éstas, producto de su idiosincrasia compleja, multidimensional y ambivalente (Broadhurst, 1982; Baron \& Hartnagel, 1996; Bouley \& Wells, 2000; Cullen et al., 2002; Indermaur \& Hough, 2002; Allen, 2004; Roberts, 2004; Hutton, 2005; Bishop, 2006; Mears et al., 2007; Fernández \& Tarancón, 2010) se encuentran moduladas por una multiplicidad de factores (Indermaur \& Hough, 2002; Satalans, 2002; Lynne \& Indermaur, 2007), lo cual 
se constata en el estudio cuando advertimos que las actitudes retributivas se encuentran mediadas por el nivel de conocimiento del sistema de justicia juvenil, la edad y la adscripción religiosa, y que este conglomerado de factores explica un 25,9\% de su varianza, de modo tal que una parte sustancial de la variabilidad de las mismas permanece en la sombra.

De conformidad con los resultados alcanzados en otros países (Lynne \& Indermaur, 2007; King \& Maruna, 2009), nuestra investigación evidencia que tales elementos constituyen predictores moderados de las actitudes punitivas. Es notorio que la provisión de información genera un impacto sobre las actitudes y la confianza pública en el sistema penal juvenil, no obstante, la correspondencia entre tales elementos sugiere no ser directa, sino transversal, arbitrada por una pluralidad de factores conexos e interdependientes (Chapman et al., 2002; Mirrless - Black, 2002; Quinton, 2011).

\section{CONCLUSIONES}

Los resultados dimanantes del experimento permiten esbozar tres conclusiones principales. En primer lugar, cabe recalcar, en consonancia con las investigaciones comparadas (Roberts, 2004; Bishop, 2006; Scott et al., 2006; Minkes, 2007), la inexistencia de datos consistentes que faculten la caracterización de la ciudadanía como punitiva o demandante de respuestas más severas hacia la delincuencia juvenil. Hallazgo que cuestiona la hipotética correspondencia y reciprocidad entre las medidas legislativas impulsadas y la voluntad pública subyacente.

En segundo término, se hace patente la incidencia de la información en la configuración de las actitudes punitivas, erigiéndose como procedimiento angular en la mitigación de las mismas, que la literatura internacional ha documentado con solidez y consistencia (Davis, 1997; Chapman et al., 2002; Hough \& Park, 2002; Mirrless Black, 2002; Gelb, 2006). No obstante, hallamos un elemento adicional que añade complejidad a esta segunda argumentación, y es el efecto de una multiplicidad de variables demográficas, personales e instrumentales sobre las primeras, que aun constituyendo predictores débiles de éstas, explican una parte sustancial de las actitudes retributivas, y que es coincidente con los estudios implementados en el escenario anglosajón (Lynne \& Indermaur, 2007; Maruna \& King, 2009; King \& Maruna, 2009).

Todo lo anterior nos conduce a trazar la última observación, constatando la naturaleza compleja, dúctil, ambivalente y multifacética de las actitudes, enunciada por 
diversos autores (Bouley \& Wells, 2000; Indermaur \& Hough, 2002; Stalans, 2002; Hutton, 2005), de acuerdo a la cual, éstas no son independientes, sino que se encuentran mediadas por una pluralidad de factores interconexos.

Futuras investigaciones habrán de analizar, más profundamente, qué subyace tras las actitudes hacia el castigo, explorando la incidencia de variables tales como el miedo al delito, la victimación vicaria o la relevancia atribuida a la temática, evaluándose, así mismo, cuál es el medio más adecuado de provisión de la información, pues tan significativo es el contenido de ésta, como la forma en que es dispensada. Del mismo modo, la aproximación empírica multimétodo podría contribuir, significativamente, en el esclarecimiento de las percepciones y posicionamientos ciudadanos, trascendiendo las limitaciones inherentes a cada uno de los procedimientos, logrando, con ello, envolver la complejidad, heterogeneidad y dinamismo consustanciales a las actitudes. Finalmente, la observancia de la persistencia en el tiempo, de las variaciones acaecidas en respuesta a la información, y su resistencia a la influencia de los medios de comunicación y los mensajes emitidos por los diversos agentes socializadores, constituye otro eje de especial trascendencia.

En definitiva, cuando la apelación a la voluntad pública constituye una de las directrices esenciales del diseño de la política criminal contemporánea, que define y subordina las respuestas ofrecidas a los menores que han transgredido las normas, el conocimiento de las actitudes públicas hacia el castigo deviene imprescindible.

\section{REFERENCIAS}

Allen, R. (2004). What works in changing public attitudes. Lessons from Rethinking Crime and Punishment. Journal for Crime, Conflict and the Media, 1(3), 55 67.

Anderson, S., Bromley, C. and Given, L. (2005). Public attitudes toward young people and youth crime in Scotland: Findings from the 2004 Scottish Social Attitudes Survey. Edinburgh: Scottish Executive Education Department.

Applegate, B.K., Cullen, F.T. and Fisher, B.S. (2002). Public views toward crime and correctional policies. Is there a gender gap? Journal of Criminal Justice, 30 (2), 89- 100.

Applegate, B.K., Davis, R.K. and Cullen, F.T. (2009). Reconsidering child saving: The extent and correlates of public support for excluding youths from the juvenile court. Crime Delinquency, Vol. 55 (1), 51 - 77.

Barber, J. and Doob, A.N. (2004). An analyses of public support for severity and proportionality in the sentencing of youthful offenders. Canadian Journal of Criminology and Criminal Justice, 46 (3), 327 - 342. 
Baron, S. and Hartnagel, T. F. (1996). "Look 'Em Up": Attitudes toward punishing juvenile offenders. Canadian Journal of Criminology and Criminal Justice, 38 (2), $191-212$.

Berger, V. (2005). Selection bias and covariate imbalances in randomized clinical trials. Chichester: John Wiley \& Sons, Ltd.

Bishop, D. M. (2006). Public opinion and juvenile justice policy: Myths and misconceptions. Criminology \& Public Policy, Vol. 5, Issue, 4, 653 - 664.

Black, T.R. (1999). Doing Quantitative Research in the Social Sciences. An Integrated Approach to Research Design, Measurement and Statistics. London: SAGE Publications.

Bouley, E.E. and Wells, T. (2000): Attitudes of citizens in a southern rural county towards juvenile crime and justice issues. Journal of Contemporary Criminal Justice, Vol. 17 (1), $60-70$.

Bravo, A., Sierra, M.J. y Del Valle, J. (2009). Evaluación de resultados de la ley de responsabilidad penal de menores. Reincidencia y factores asociados. Psicothema, Vol. 21, No. 4, 615 - 621 .

Broadhurst, R.G. (1982). Public opinion, punishment and crime. Some notes on a survey approach. Australia: University Extension.

Byrne, D. (2002). Interpreting Quantitative Data. London: SAGE Publications.

Capdevilla, M., Ferrer, M. y Luque, E. (2005). La reincidencia en el delito en la justicia de menores. Centre d’Estudis Juridics i Formació Especialitzada. Generalitat de Catalunya.

Chapman, B., Mirrlees - Black, C. and Brawn, C. (2002). Improving public attitudes to the Criminal Justice System: The impact of information. Home Office Resarch Study, 245. London: Home Office.

Clarck - Carter, D. (2002). Investigación cuantitativa en psicología. Del diseño experimental al reporte de investigación. México: Oxford University Press.

Cohen, J. (1998). Statistical power analysis for the behavioral sciences. New York: Academic.

Cullen, F., Evans, S., Scott, J. and Burton, V. (1990). Public support for correctional treatment: The tenacity of rehabilitative ideology. Criminal Justice Behavior, Vol. 17, 6-18.

Cullen, F., Fisher, B. and Applegate, B. (2000). Public Opinion about Punishment and Corrections. In Crime and Justice: A review of Research. Chicago: University of Chicago. Vol. 27, 1 - 79.

Cullen, F., Pealer, J.A., Fisher, B.S., Applegate, B.K. \& Santana, S.A. (2002). Public support for correctional rehabilitation in America: Change or consistency? In J. Roberts and M. Hough (Eds.) Changing attitudes to punishment. Public opinion, crime and Justice. Oregon: Willan Publishing.

Davis, D.W. (1997). Attitudes toward crime and criminal justice: What you find depends on what you ask. Institute for Public Policy \& Social Research, Michigan State University.

Díez - Ripollés, J.L. (2003). La racionalidad de las leyes penales. Práctica y teoría. Madrid: Editorial Trotta.

Doble, J. (2002). Attitudes to punishment in the US. In J. Roberts and M. Hough (Eds.) Changing attitudes to punishment. Public opinion, crime and justice. Oregon: Willan Publishing. 
Doob, A.N. (2000). Transforming the punishment environment: Understanding public views of what should be accomplished at sentencing. Canadian Journal of Criminology and Criminal Justice, 42 (3), 323 - 340.

Escobar, G. y Varona, D. (2007). Actituds dels catalans envers les sancions penals. Catalunya: Generalitat de Catalunya.

Fernández, E., Bartolomé, R., Rechea, C. y Megías, A. (2009). Evolución y tendencias de la delincuencia juvenil en España. Revista Española de Investigación Criminológica, No. 7.

Fernández, E. y Tarancón, P. (2010). Populismo punitivo y delincuencia juvenil: Mito o realidad. Revista Electrónica de Ciencia Penal y Criminología, 12 - 08.

García, M., Ibáñez, J. y Alvira, F. (Comp.) (1996). El análisis de la realidad social. Métodos y técnicas de investigación. Madrid: Alianza Editorial.

García, M.D., Martín, E., Torbey, A. y Rodríguez, C. (2010). La valoración social de la Ley de Responsabilidad Penal de los Menores. Psicothema, Vol. 22, No.4, 865 $-871$.

Gelb, K. (2006). Myths and Misconceptions: Public opinion versus public judgment about sentencing. Melbourne: Sentencing Advisory Council.

Goidel, R. K., Freeman, C. M. and Procopio, S. T. (2006). The impact of television viewing on perceptions of juvenile crime. Journal of Broadcasting \& Electronic Media, 50, 119 - 139.

Green, D. (2006). Public opinion versus public judgment about crime. British Journal of Criminology, No. 46, $131-154$.

Grinnell, R.M. (1997): Social Work Research \& Evaluation. Quantitative and Qualitative Approaches. United States of America: F.E. Peacock Publishers, Inc.

Guetti, S. and Redlich, A.D. (2001). Reactions to youth crime: perceptions of accountability and competency. Behavioral Sciences and the Law, Vol. 19 (1), $33-52$.

Haines, A. (2007). Juvenile crime and punishment in Bucharest, Romania: A public opinion survey. Internet Journal of Criminology, $1-19$.

Halsey, K. and White, R. (2008). Young people, Crime and Public Perceptions: A review of the literature. LGA Research Report. Slouh: NFER.

Heppner, P.P., Kivlighan, D.M. and Wampold, B.E. (2008). Research design in counseling. Belmont: Thompson.

Hough, M. and Park, A. (2002). Attitudes to crime and punishment: the results of a deliberative poll of public opinion. London: Esmée Fairbairn Foundation.

Hough, M. and Roberts, J.V. (1998). Attitudes to punishment: Findings from the British Crime Survey. Home Office Research Study, No. 179. London: Home Office.

Hough, M. and Roberts, J.V. (2004). Youth crime and youth justice: Public opinion in England and Wales. Bristol: Policy Press.

Hutton, N. (2005). Beyond populist punitiveness? Punishment and Society, Vol. 7 (3), $243-258$.

Indermaur, D. \& Hough, M. (2002). Strategies for changing public attitudes to punishment. In J. Roberts and M. Hough (Eds.) Changing attitudes to punishment. Public opinion, crime and justice. Oregon: Willan Publishing.

Indermaur, D. (2009). What can we do to engender a more rational and less punitive crime policy? European Journal of Criminal Policy and Research, No. 15, 181 $-199$. 
Jackson, S.L. (2009). Research methods and statistics: A critical thinking approach. Belmont: Wadsworth.

King, A. and Maruna, S. (2009). Is a conservative just a liberal who has been mugged? Exploring the origins of punitive views. Punishment \& Society, Vol. 11 (2), $147-169$.

Krisberg, B. and Marchionna, S. (2007). Attitudes of US voters toward youth crime and the justice system. National Council on Crime and Delinquency, Oakland.

Kury, H., Obergfell - Fucks, J. and Smartt, U (2002). The evolution of public attitudes to punishment in Western and Eastern Europe. In J. Roberts and M. Hough (Eds.) Changing attitudes to punishment. Public opinion, crime and justice. Oregon: Willan Publishing.

Larrauri, E. (2005). Populismo punitivo y penas alternativas a la prisión. En S. Bacigalupo y M. Cancio (Comp.), Derecho penal y política transnacional. Barcelona: Atelier.

Lynne, D.R. and Indermaur, D. (2007). Predicting punitive attitudes in Australia. Psychiatry, Psychology and Law, 14, 1, 56 - 65.

Marteache, N., Martínez, M. y Pérez, M. (2010). Comparación entre opinión pública y "opinión pública meditada" en relación a la delincuencia sexual. Revista Española de Investigación Criminológica, Artículo 7, № 8.

Maruna, S. y King, A. (2009). Once a criminal, always a criminal?: "Redeemability" and the psychology of punitive public attitudes. European Journal of Criminal Policy and Research, No. 15, 7 - 24.

Mateo, M.J. y García, M. (1993). Estadística aplicada a las ciencias sociales. Madrid: Universidad Nacional de Educación a Distancia.

Matthews, R. (2005). The myth of punitiveness. Theoretical Criminology, Vol. 9 (2), $175-201$.

Mayhew, P. and Van Kesteren, J. (2002). Cross - national attitudes to punishment. In J. Roberts and M. Hough (Eds.) Changing attitudes to punishment. Public opinion, crime and justice. Oregon: Willan Publishing.

Mears, D., Hay, C., Gertz, M. and Mancini, C. (2007). Public opinion and the foundation of the Juvenile Court. Criminology, Vol. 25, No. 1, 223 - 257.

Medina, J.J. (2003). Inseguridad ciudadana, miedo al delito y policía en España. Revista Electrónica de Ciencia Penal y Criminología, No. 5 (03).

Minkes, J. (2007). Change, continuity and public opinion in youth justice. International Criminal Justice Review, Vol. 17, No. 4, 340 - 349.

Mirless - Black, C. (2002). Improving public knowledge about crime and punishment. In J. Roberts and M. Hough (Eds.) Changing attitudes to punishment. Public opinion, crime and justice. Oregon: Willan Publishing.

Nacro Youth Crime (2001) Public opinion and youth Justice. Youth Crime Briefing, 1-8.

Nagin, D.S., Piquero, A.R., Scott, E.S. and Steinberg, L. (2006). Public preferences for rehabilitation versus incarceration of juvenile offenders: Evidence from a contingent valuation survey. Crime and Public Policy, Vol. 5, No. 4, 301 326.

Punch, K.F. (2001). Introduction to Social Research. Quantitative \& Qualitative Approaches. London: SAGE Publications.

Quinton, P. (2011). The impact of information about crime and policing on public perceptions. The results of a randomized controlled trial. UK: National Policing Improvement Agency. 
Rechea, C., Fernández, E. y Benítez, M.J. (2004). Tendencias sociales y delincuencia. Centro de Investigación en Criminología, Universidad de Castilla - La Mancha, Informe No. 11.

Ripolles, J.J. y García, E. (Eds.) (2009). Encuesta a víctimas en España. Málaga: Instituto Andaluz Interuniversitario de Criminología.

Roberts, J.V. (1992). Public opinion, crime and criminal justice. Crime and justice: A review of research, Vol. 16, $99-180$.

Roberts, J. (2002). Public opinion and the nature of community penalties: international findings. In J. Roberts and M. Hough (Eds.) Changing attitudes to punishment. Public opinion, crime and justice. Oregon: Willan Publishing.

Roberts, J. and Hough, M. (2002). Public attitudes to punishment: The context. In J. Roberts and M. Hough (Eds.) Changing attitudes to punishment. Public opinion, crime and justice. Oregon: Willan Publishing.

Roberts, J. (2004): Public Opinion and Youth Justice. Crime \& Justice: A review of Research, Vol. 31, 495: 542.

Rubio, M.J., y Varas, J. (2004). El análisis de la realidad en la intervención social. Métodos y técnicas de investigación. Madrid: Editorial CCS.

Salisbury, H. (2004). Public attitudes to the criminal justice system: The impact of providing information to British Crime Survey respondents. Home Office. London: Report 64/04.

San Juan, C. y Ocáriz, E. (2009). Evaluación de la intervención educativa y análisis de la reincidencia en la Justicia de Menores en la CAPV. Vitoria/Gasteiz: Gobierno Vasco

Scott, E. S., Repucci, N. D., Antonishak, J. and Degennaro, J. T. (2006). Public attitudes about the culpability and punishment of young offenders. Behavioral Sciences and the Law, No. 24 (6), $815-832$.

Singer, L. and Cooper, S. (2008). Inform, persuade and remind. An evaluation of a project to improve public confidence in the criminal justice system. London: Ministry of Justice.

Sprott, J. B. (1996). Understanding public views of youth crime and the youth justice system. Canadian Journal of Criminology, 38 (3), 271 - 290.

Stalans, L.J. (2002). Measuring attitudes to sentencing. In J. Roberts and M. Hough (Eds) Changing attitudes to punishment: Public opinion, crime and justice. Cullompton: William Publishing, pp. 15 - 32.

Stead, M., MacFadyen, L. and Hasting, G. (2000). What do the public really feel about non - custodial penalties? London: Esmée Fairbairn Foundation.

Toharia, J.J. (2002). Las encuestas de opinión y las decisiones políticas: El caso de la evaluación y reforma de la justicia. Revista Española de Investigaciones Sociológicas, $\mathrm{N}^{\circ} 99,223-235$.

Varma, K.N. (2006). Face - ing the offender: Examining public attitudes toward Young offenders. Contemporary Justice Review, Vol. 9, No. 2, 175 - 187.

Varona, D. (2008). Ciudadanos y actitudes punitivas: Un estudio piloto de población universitaria española. Revista Española de Investigación Criminológica, $N^{o} 6$.

$\mathrm{Wu}$, B. (2000). Determinants of public opinion toward juvenile waiver decision. Juvenile and Family Court Journal, Vol. 50, 9 - 20. 